\title{
Changes of IL-1, TNF-Alpha, IL-12 and IL-10 Levels with Chronic Liver Failure
}

\author{
Guowang Liü, Kecheng Tang, Qian Li, Guiyu Yuan, Wukui Cao, Wei Lu* \\ Physician-in-Charge Tianjin Infectious Disease Hospital, Tianjin, China \\ E-mail: luwei1966@126.com, regulaly@163.com \\ Received January 6, 2011; revised January 25, 2011; accepted February 11, 2011
}

\begin{abstract}
[Aim] To investigate the action of cytokines in patients with chronic liver failure and to explore the roles of cytokines in chronic liver failure. [Methods] Thirty-one patients with chronic liver failure admitted from May 2006 to November 2009 were included. Thirty cases with mild to moderate chronic type B hepatitis admitted concomitantly were regarded as control. IL-1, TNF-alpha, IL-12, IL-10 Levels in serum was the factors to determine at clinical diagnosis and 2-week treatment. We analyzed levels of cytokines action in 31 chronic liver failure patients both at clinical diagnosis and 2-week treatment in comparison with control group. [Results] We analyzed IL-1 level in death group at clinical diagnosis and 2-week treatment were significant higher than in survival and control groups, furthermore it was also higher in survival group than in control group. At clinical diagnosis, TNF-alpha level in death group was higher than that in control. However, there was no obviously difference between the death group and the survival group in TNF-alpha level. With the progression of the disease, TNF-alpha level was remarkably risen in death group, but decreased in survival group. IL-10 level was dramatically increased in death group, but no changed in survival group. IL-12 level in death group was higher than in survival group, but lower than in control group. [Conclusions] The levels of TNF-alpha, IL-1, IL-10 and IL-12 in patients with chronic liver failure was increased and the increase of IL-10 is secondary to elevation of IL-12.
\end{abstract}

Keywords: Chronic Liver Failure, Cytokine, Hepatitis B

The pathogenesis of liver failure is very complicated, which involves virus-induced primary immuno-pathological damage, as well as cytokine-mediated effect. Liver failure and multiple organ failure accompany with the level changed in cytokines [1]. Each cytokine factor has its own physiological function and interacts to each others, which is also complicated to understand their roles. Anti-inflammatory is the IL-10 main function. IL-1, TNF- $\alpha$ are both inflammatory mediators. IL-12 has multiple biological activities, including anti-viral, anti-tumor and immunoregulation [2]. To an extent, IL-12 reflects capabilities of human body in killing viruses. In this study, we investigated the levels of IL-1, TNF-alpha, IL-12 and IL-10 in serum in order to explore the roles of cytokines in pathogenesis and progression of chronic severe hepatitis.

\footnotetext{
\#Bachelor in Medicine, physician-in-charge Tianjin Infectious Disease Hospital 300192 E-mail: regulaly@163.com

${ }^{*}$ Corresponding author, Doctor of Philosophy in Medicine, professor Tianjin Infectious Disease Hospital 300192

E-mail: luwei1966@126.com
}

\section{Subjects and Methods}

\subsection{Patients in this Study}

Thirty-one patients ( 24 males, 7 females, average age of $40.08 \pm 10.06$ years, range $22-65$ years) with chronic liver failure admitted from May 2006 to November 2009 were included. Based on prognostic situation after one month treatment, these patients were divided into two groups: survival group and death group(survival group, 14 males, 4 females, average age of $39.22 \pm 7.17$ years, range 30-55 years; death group, 10 males, 3 females, average age of $41.23 \pm 13.36$ years, range $22-65$ years). All patients had hepatitis B virus infected, eleven cases were in early stage and 20 cases in middle stage.

The other thirty cases with mild to moderate chronic type B hepatitis admitted concomitantly were regarded as control. This series contained 23 males and 7 females, with the mean age of $40.28 \pm 11.29$ years (range 19-66 
years). No combined infection with other hepatitis viruses were detected. Diagnosis were referred to the revised "Diagnostic and treatment guidelines for liver failure" by Liver Failure and Artficial Liver Group ,Chinese Society of Infectious Diseases, Chinese Medical Association; Severe Liver Diseases and Artifcial Liver Group, Chinese Society of Hepatology, Chinese Medical Association [3]. There were no statistical differences in age, sex ratio of number of early to middle stage patients among survival group, death group and control group.

\subsection{Therapy Methodologies}

All chronic liver failure patients had to rest in bed and had light diet. Combined therapy with hepatocyte growth-promoting factors, sodium tanshinon IIA silate and alprostadil were adopted. 22 cases in hepatic failure group underwent non-bioartificial liver therapy including plasma exchange and hemofiltration. Chronic hepatitis patients had glutathione and licorice root preparation.

\subsection{Observed Items}

Cytokines: IL-1, TNF-alpha, IL-12, IL-10.

Blood samples were collected in the morning from empty stomach clinical diagnosis and 2-week treatment patients. Levels of serum IL-1, TNF-alpha, IL-12, IL-10 were measured by Luminex-100 bead-based fluorescence flow cytometer (Luminex-100). Reagents come from Becton, Dickinson and Company(Anti-XBP-1). Artificial liver therapy was not taken in 24 hours before blood collection.

\subsection{Statistical Analysis Method}

Data were analyzed by SPSS11.5 (Analysis of Variance).

\section{Results}

\subsection{Comparison of Cytokine Levels at Clinical Diagnosis Stage (Table 1)}

IL-1, IL-10 and TNF- $\alpha$ level in death group was signifycantly higher than in the other two groups, and IL-1 level in survival group was higher than that in control group both at clinical diagnosis stage. IL-12 level in control group was the lowest, and it was the highest in death group at clinical diagnosis stage. IL-1, IL-12 levels were differences among those three groups. TNF- $\alpha$ level in control group was different from the other two groups and no statistical differences between the other two groups. IL-10 level in death group was different with the other two groups and no statistical differences between the other two groups.

\subsection{Comparison of Cytokine Levels at 2-Week of Treatment Stage (Table 2)}

IL-1, IL-10 and TNF- $\alpha$ levels in death group was significantly higher than in the other two groups, and IL-1

Table 1. Comparison of cytokine levels $(\mathrm{pg} / \mathrm{ml})$ at clinical diagnosis (mean $\pm \mathrm{SD})$.

\begin{tabular}{cccccc}
\hline Items & Survival group (18) & Death group (13) & Control group (30) & $F$ & $P$ \\
\hline IL-1 & $86.00 \pm 26.38^{*}$ & $151.46 \pm 52.89^{*}$ & $40.96 \pm 18.10^{*}$ & 59.38 & .000 \\
TNF- $\alpha$ & $53.50 \pm 73.49$ & $87.76 \pm 91.38$ & $14.80 \pm 4.74 \#$ & 7.796 & .001 \\
IL-12 & $217.16 \pm 70.97^{*}$ & $289.38 \pm 89.17^{*}$ & $45.20 \pm 20.06^{*}$ & 99.767 & .000 \\
IL-10 & $28.91 \pm 16.75$ & $247.46 \pm 192.05 \#$ & $27.20 \pm 14.06$ & 31.578 & .000 \\
\hline
\end{tabular}

Data was analyzed by q test. *: difference among in those three groups, \#: difference with the other two groups, and no statistical difference between the other two groups.

Table 2. Comparison of cytokine levels $(\mathrm{pg} / \mathrm{ml})$ at 2-week treatment (mean $\pm \mathrm{SD})$.

\begin{tabular}{cccccc}
\hline Items & Survival group (18) & Death group (13) & Control group (30) & $F$ & $P$ \\
\hline IL-1 & $64.61 \pm 21.19^{*}$ & $82.14 \pm 20.85^{*}$ & $26.63 \pm 7.17^{*}$ & 58.956 & 0.000 \\
TNF- $\alpha$ & $28.88 \pm 26.20$ & $283.92 \pm 586.06 \#$ & $13.60 \pm 4.41$ & 5.397 & 0.007 \\
IL-12 & $175.33 \pm 57.08^{*}$ & $239.02 \pm 56.02^{*}$ & $31.23 \pm 17.39^{*}$ & 118.42 & 0.000 \\
IL-10 & $49.88 \pm 157.46$ & $346.50 \pm 313.71 \#$ & $18.56 \pm 8.12$ & 24.927 & 0.000 \\
\hline
\end{tabular}

Data was analyzed by q test. *: difference among the three groups, \#: difference from the other two groups, and no statistical difference between the other two groups. 
level in survival group was higher than in control group. IL-12 level in control group was the lowest, and it was the highest in death group after 2-week treatment. IL-1, IL-12 level are difference among those three groups. TNF- $\alpha$ and IL-10 level in death group were different from the other two groups and no statistical difference between the other two groups. IL-1 level at clinical diagnosis stage was higher than after 2 -week treatment, both in death group and survival group.

\section{Discussion}

IL-1 helps body to fight against pathogenic microorganisms and it is also one of non-specific defensive factors. The results of at clinical diagnosis and 2-week treatment stages were shown IL-1 level in death group was signifycantly higher than in the other two groups meanwhile, it was the lowest in control group both at clinical diagnosis and 2-week treatment stages, which suggested IL-1 might reflect the degree of inflammatory reaction and serve as important index for prognosis of severe hepatitis. Nevertheless, with the progression of the disease, IL-1 level at clinical diagnosis stage was higher than after 2-week treatment, both in death group and survival group. This result implied that IL-1 secretion was decreasing while the progression of Liver failure, therefore, resultes in compromising of immunity. Those effectors, the possible reason, could be generated by a number of immunocytes upon activation in early stage of disease, whereas those effectors were decreasing indirectly due to the apoptosis of immunocytes in the last stage.

TNF- $\alpha$ has a variety of biological effects, it is the important factor in causing liver damage [4]. The level of TNF- $\alpha$ was not only reflecting the capability of resisting viral infection, but also associated with the immunopathological to damage hepatocytes. At clinical diagnosis stage, TNF- $\alpha$ level in severe hepatitis patients was significantly higher than in control group, whereas there was no statistical differences between death group and survival group. With disease progressing, TNF- $\alpha$ level was remarkably increasing in death group but decreasing in survival group. After 2-week treatment, it was obviously higher in death group than in survival group. It was suggested that changes of TNF- $\alpha$ could be the index of the prognostic assessment, and TNF- $\alpha$ also reflected the inflammation severity. Hepatic necrosis was reflected by excessively high level of TNF-alpha, it might be one of the important reasons of death.

IL-10, an important anti-inflammatory cytokine, participated in principal negative feedback regulation including inhibiting the generation of the inflammatory factor and the colony stimulating factor in one hand and suppressed the anti-viral immunity of the body in the other hand. In this study, it shown anti-inflammatory cytokines in severe hepatitis patients of death group were dramatically raise, but not significantly changed in survival group, which suggested the increasing of anti-inflammatory cytokines in severe hepatitis patients especially at the last stage was an indirect indicator of severe inflammation. And the raise of cytokine level could be the result in immunity decreasing. The immunity of patients who suffered from severe hepatitis might be varied at different stages of disease. At early stage, the inflamematory cytokines were releasing, and there is no increasing in non-inflammatory factors. If the disease had been well controlled, anti-inflammatory factors would be no longer increasing. Otherwise, they would be signifycantly increasing to prevent the body from excessive immune attack. Simultaneously, the immunity of patients was declined.

IL-12 level in death group was higher than in survival group both at clinical diagnosis and 2-week treatment stage, and it was the lowest factor in control group, which suggested there was intensive reaction for eliminating pathogen at beginning of severe hepatitis, especially in heavy disease patients.

IL-1 could be amplified by the biological effect of TNF- $\alpha$, which could aggravate hepatic necrosis. Under normal physiological condition, TNF- $\alpha$ and IL-1 could be cleared by the liver. However, in case of liver failure, endotoxin could activate macrophages, which could impair the liver's capability of eliminating cytokines. Thus, TNF- $\alpha$ and IL-1 were the directly factors to develop of liver failure. In our study, IL-10 raising did not suppress the IL-12 increasing. In this study, it had shown IL-10 raising might be caused by increasing of IL-12, it might be a compensatory response, which might prevent body from excessive immunological damage. In addition, the results of IL-10 in our study was different from Yumoto $\mathrm{E}$ results [5]. We need more samples for the further study.

\section{References}

[1] H. Isoniemi, A. M. Koivusalo, H. Repo and et a1. "The Effect of Albumin Dialysis on Cytokine Levels in Acute Liver Failure and Need for Liver Transplantation," Transplantation Proceedings, Vol. 37, No. 2, 2005, pp. 1088-1090. doi:10.1016/j.transproceed.2004.11.060

[2] Tao Wen and Hao Wu, "Research Progress of Interleukin-12 and Its Application in Management of HIV Infection," Foreign Medical Sciences (Epidemiology Lemology Fascicle), Vol. 30, No. 5, 2003, pp. 271-274.

[3] J. Clin Hepatol, "Diagnostic and treatment guidelines for liver failure," Vol. 9, No. 6, 2006.

[4] R. F. Schwabe and D. A. Brenner, "Mechanisms of Liver Injury Tnf-Alpha-Induced Liver Injury: Role of IKK, 
JNK, and ROS Pathways," American Journal of Physiology - Gastrointestinal and Liver Physiology, Vol. 290, No. 4, 2006, pp. 583-589. doi:10.1152/ajpgi.00422.2005

[5] E. Yumoto, T. Higashi and K. Nouso, "Serum GammaInterferon-Inducing Factor (Il-18) and IL-10 Levels in
Patients with Acute Hepatitis and Fulminant Hepatic Failure," Journal of Gastroenterology and Hepatology, Vol. 17, 2002, pp. 285-294.

doi:10.1046/j.1440-1746.2002.02690.x 